\title{
Cumplimiento de las medidas de prevención de factores de riesgo vascular en pacientes ingresados con ictus agudo. Análisis de un registro multicéntrico: registro EPICES (III)
}

Miguel Blanco, José Vivancos-Mora, José Castillo, en nombre de los investigadores del registro EPICES

Introducción. Medidas farmacológicas, dietéticas y de estilos de vida para controlar los factores de riesgo son eficaces para prevenir la enfermedad cerebrovascular; sin embargo, su implementación en la clínica no parece óptima.

Objetivo. Identificar el cumplimiento de medidas de prevención en una muestra hospitalaria de 6.197 pacientes hospitalizados con ictus atendidos por neurólogos.

Pacientes y métodos. Análisis de un objetivo secundario del registro EPICES. Se consideró prevención primaria en los pacientes sin antecedentes previos de ataque isquémico transitorio, ictus, enfermedad coronaria o enfermedad arterial periférica $(n=3.977)$; prevención secundaria, en pacientes con antecedentes de enfermedad coronaria o arterial periférica, pero sin antecedentes de enfermedad cerebrovascular $(n=1.047)$; y prevención secundaria neurológica, en pacientes con antecedentes de ataque isquémico transitorio o ictus, independientemente de que hubiesen presentado enfermedad coronaria 0 arterial periférica $(n=1.173)$.

Resultados. La prevención secundaria y secundaria neurológica fue más eficaz que la prevención primaria $(p=0,028$ y $p<0,0001$, respectivamente). El control fue superior en los centros con algún tipo de estructura asistencial para la atención de los pacientes con ictus $(p<0,0001)$. La influencia de la edad en las decisiones terapéuticas siguió un patrón similar en los tres tipos de prevención, con una disminución significativa de la anticoagulación en los pacientes con más de 80 años.

Conclusión. El registro EPICES confirma el mal control de los factores de riesgo cerebrovascular. Globalmente, se demuestra que los objetivos que implican la modificación de los estilos de vida se cumplimentan peor que las medidas preventivas farmacológicas.

Palabras clave. Enfermedad cerebrovascular. Factores de riesgo. Prevención primaria. Prevención secundaria.

\section{Introducción}

El conjunto de medidas farmacológicas, dietéticas y de estilo de vida para controlar los factores de riesgo de enfermedades cerebrovasculares han demostrado una eficacia, en términos de mortalidad y morbilidad, sin duda superior a la alcanzada por la suma de todos los procedimientos terapéuticos utilizados en la fase aguda de la enfermedad [1,2]. Sin embargo, su implementación en la práctica clínica habitual dista mucho de resultar óptima, aunque los resultados reflejados en la bibliografía son dispares [3-10].

A pesar de que parece comprobado que la modificación de estilos de vida con riesgo cerebrovascular es más eficaz que la introducción de fármacos [11], su implementación en la clínica es considerablemente menor [10]. Las guías recomiendan iniciar lo antes posible la prevención primaria y, en el caso de la prevención secundaria, debe instaurarse durante el tiempo de hospitalización [5]. Las intervenciones multifactoriales, farmacológicas, dietéticas y de estilos de vida son notablemente superiores a intervenciones aisladas sobre un solo factor de riesgo [12-14]. Todo este conjunto de conocimientos contrasta con el resultado de estudios que demuestran que el tiempo dedicado por los médicos a explicar las ventajas de las medidas de prevención es insuficiente; el $50 \%$ de los pacientes con ictus no recibe ningún consejo dietético [15] y un 54\% no es informado sobre las necesarias modificaciones del estilo de vida previo $[5,16]$. Aunque se conoce la influencia que el medio familiar ejerce sobre los estilos de vida de los pacientes, tampoco es el objetivo de intervenciones específicas por parte de los médicos [17]. El presente estudio intenta identificar el grado de cumplimiento de las medidas de prevención primaria y secundaria en una extensa muestra
Servicio de Neurología; Instituto de Investigaciones Sanitarias de Santiago, IDIS; Hospital Clínico Universitario; Universidad de Santiago de Compostela; Santiago de Compostela, A Coruña (M. Blanco, J. Castillo). Servicio de Neurología; Instituto de Investigación Sanitaria Princesa; Hospital Universitario La Princesa; Madrid, España (J. Vivancos-Mora).

Correspondencia: Dr. José Castillo Sánchez. Servicio de Neurología. Hospital Clínico Universitario de Santiago. Travesa da Choupana, s/n. E-15706 Santiago de Compostela (A Coruña).

E-mail:

jose.castillo@usc.es

Declaración de intereses: El registro EPICES fue patrocinado por Pfizer España. J.C. y J.V.M. recibieron honorarios por la dirección del registro.

Aceptado tras revisión externa: 10.04.12.

Cómo citar este artículo: Blanco M, Vivancos-Mora J, Castillo J, en nombre de los investigadores del registro EPICES. Cumplimiento de las medidas de prevención de factores de riesgo vascular en pacientes ingresados con ictus agudo. Análisis de un registro multicéntrico: registro EPICES (III). Rev Neurol 2012; 54: 523-9.

Investigadores participantes en el registro EPICES:

J. Abella-Corral, M.J. Abenza-Abildua, M. Alonso de Leciñana, J. ÁlvarezGutiérrez, J. Álvarez-Sabín, C. AntónGonzález, J. Arenillas-Lara, M. AriasRodríguez, A. Arjona-Padillo, C. Barrero-Ramírez, A. BarrosoMerinero, L. Benavente-Fernández, L. Berenguer-Ruiz, Y. BravoAnguiano, D. Cánovas-Verge, P. Carbonell-Corvillo, A.M. CarraVélez, I. Casado-Menéndez,

I. Casado-Naranjo, L. Castilla-Guerra, 
J. Castillo-Sánchez, C. Cid-Rodríguez, M.J. Cruz-Huertas, P. de JuanHernández, C. Díaz-Marín, F. DíazOtero, E. Díez-Tejedor, F. DomínguezSanz, J. Egido-Herrero, M.I. Escalza-Cortina, E. Escolar-Escamilla J. Estela-Herrero, J. Fernández-Ferro, E. Franquet-Gómez, R. GalianoBlancart, J. Gallego-Cullere, J.C.

García-Moncó, A. García-Pastor, L. García-Tuñón, A. Gil-Núñez, J.C. Giner-Bernabeu, S. GomaraLópez, A. Gómez Díaz-Castroverde C. Gómez-Escalonilla, J.C. GómezSánchez, C. González-Rodríguez, J. Gutiérrez-García, R. HernándezClares, J.J. Hernández-Regadera, L. Hernández-Rubio, M.E. HerreroPrieto, G. Izquierdo-Ayuso M.C. Jiménez-Martínez, M. JiménezNieto, M. Lezcano-Rodas, J.I. LópezGastón, J.F. Maestre-Moreno, C.G. Marrero-Falcón, J. Martí-Fábregas, S. Martín-Balbuena, M.M. MartínezMartínez, E. Martínez-Vila, A. MartínezYélamos, J. Masjuán-Pallejo, V. MecaLallana, J.P. Medina-Báez, A. MedinaRodríguez, M.V. Mejías-Olmedo,

E. Meneu-García, M. Millán-Torne C. Monforte-Dupret, A. MoralPijaume, J. Muñoz-Torrero Rodríguez C. Naranjo-Fernández, J.J.OchoaSepúlveda, J. Oliva-Navarro,

E. Orts-Castro, E. Palacio-Portilla,

E. Palomeras-Soler, R. Pego-Reigosa, M.C. Peinado-Cantero, J. PeñaMartínez, C. Pérez, N. Pérez de la Ossa-Herrero, C. Pérez-Lázaro, R.

Piñeiro-Bolaño, M.A. Pons-García, A. Ponz de Tienda, F. Purroy García, J.M. Ramírez-Moreno,

M. Rebollo Álvarez-Amandi, A. ReyPérez, G. Ruiz-Ares, J. SanahujaMontesinos, O. Sánchez del Valle

J. Sánchez-Herrero, C. Sánchez-

Sánchez, J. Saura-Salvado, T. Segura-

Martán, M. Seijo-Martínez, M.C.

Serrano-Munuera, M. Serrano-Ponz,

J. Tejada-García, T. Tortosa-Sánchez,

J. Trejo-Gabriel y Galán, P. Vázquez-

Alen, I. Villegas-Martínez, J. VivancosMora, A. Yusta-Izquierdo. hospitalaria de pacientes con ictus atendidos por neurólogos en 66 centros públicos españoles.

\section{Pacientes y métodos}

La determinación de la prevalencia de los factores de riesgo vascular y del grado de cumplimiento de las medidas de prevención primaria y secundaria fue un objetivo secundario del registro EPICES. El análisis de la prevalencia de los factores de riesgo vascular en los distintos tipos de ictus se publicó previamente [18].

La descripción de la metodología utilizada para la elaboración del registro EPICES ya se realizó [18, 19]; se trata de un registro observacional y prospectivo de pacientes ingresados por ictus agudo a cargo de neurólogos.

También se publicaron las definiciones utilizadas prospectivamente de los tipos de ictus y de los factores de riesgo [18]. Los criterios de buen control de los factores de riesgo vascular utilizados en el registro EPICES fueron los siguientes: para la tensión arterial se exigía que la mayoría $(\geq 60 \%$ ) de las medidas determinadas estuviera en el rango de los objetivos terapéuticos según el grupo de riesgo del paciente o, en su defecto, por debajo de 140/90 $\mathrm{mmHg}$, al menos en los últimos tres meses. El buen control de la diabetes implica que la mayoría $(\geq 60 \%)$ de las determinaciones de glucemia en sangre venosa estuviera por debajo de $126 \mathrm{mg} / \mathrm{dL}$ (7 mM/L), al menos en los últimos tres meses. Para la dislipemia, que la mayoría ( $\geq 60 \%$ ) de las determinaciones de colesterol total y colesterol LDL se mantuviesen en el rango de los objetivos terapéuticos según el grupo de riesgo del paciente o, en su defecto, por debajo de $190 \mathrm{mg} / \mathrm{dL}$ (colesterol total) y $115 \mathrm{mg} / \mathrm{dL}$ (colesterol LDL). El buen control del tabaquismo y del enolismo exigía el abandono real de los hábitos tóxicos. La consideración del buen control de la obesidad implicó una disminución $\geq 10 \%$ del peso corporal previo, y para el abandono del sedentarismo se valoró un cambio del hábito reconocido por el paciente y sus familiares. En el cuaderno de recogida de datos se registró la percepción del neurólogo sobre el control de los factores de riesgo vascular.

En una publicación previa [19] se definieron los criterios utilizados para la acreditación de los equipos y unidades de ictus, así como la consideración de la existencia de guardias de neurología. Para el análisis utilizado en este trabajo, la existencia de equipos, unidades o guardias de neurología se agrupó post hoc en alguna estructura asistencial para la atención de los pacientes con ictus.
Para la identificación del grado de cumplimentación de las medidas de prevención, hemos definido prospectivamente tres grupos:

- Prevención primaria: en pacientes sin antecedentes previos de ataques isquémicos transitorios, ictus, enfermedad coronaria o enfermedad arterial periférica diagnosticados.

- Prevención secundaria: en pacientes con antecedentes de enfermedad coronaria o enfermedad arterial periférica, pero sin antecedentes de ataque isquémico transitorio o ictus.

- Prevención secundaria neurológica: en pacientes con antecedentes previos de ataques isquémicos transitorios o ictus, independientemente de que hubiesen presentado enfermedad coronaria o enfermedad arterial periférica.

El análisis estadístico se efectuó en todos los pacientes que cumplieron todos los criterios de inclusión y ninguno de los criterios de exclusión [18]. El análisis descriptivo sólo implicó variables cualitativas y se expresó como porcentajes, y para su comparación se utilizó el test de $\chi^{2}$. Para el análisis se utilizó el programa estadístico SPSS v. 16.0.

\section{Resultados}

El registro EPICES incluyó 6.280 pacientes de 66 hospitales públicos españoles, de los cuales 6.197 fueron válidos para el análisis del grado de cumplimiento de las medidas de prevención. En el grupo de prevención primaria se incluyeron 3.977 pacientes, 1.047 en el de prevención secundaria y 1.173 en el de prevención secundaria con enfermedad cerebrovascular previa.

La identificación de los factores de riesgo vascular fue similar en los tres grupos de pacientes, con excepción de la dislipemia, que se detectó en un mayor número de pacientes en prevención secundaria $(p=0,043)$. Sin embargo, en la implementación de las medidas de prevención se detectaron diferencias significativas; el porcentaje de la administración de estatinas y de medidas activas contra el tabaquismo y contra el sedentarismo fue mayor en los pacientes en prevención secundaria, mientras que la administración de anticoagulantes en pacientes con fibrilación auricular y de antihipertensivos fue superior en los pacientes con enfermedad cerebrovascular previa (Tabla I). El número de pacientes sin fibrilación auricular que recibieron tratamiento antiagregante plaquetario fue similar en la prevención secundaria, sin y con antecedentes de enfermedad cerebrovascular previa, y en ambos 
Figura. Porcentaje de pacientes que reciben tratamientos farmacológicos preventivos en relación con la edad.
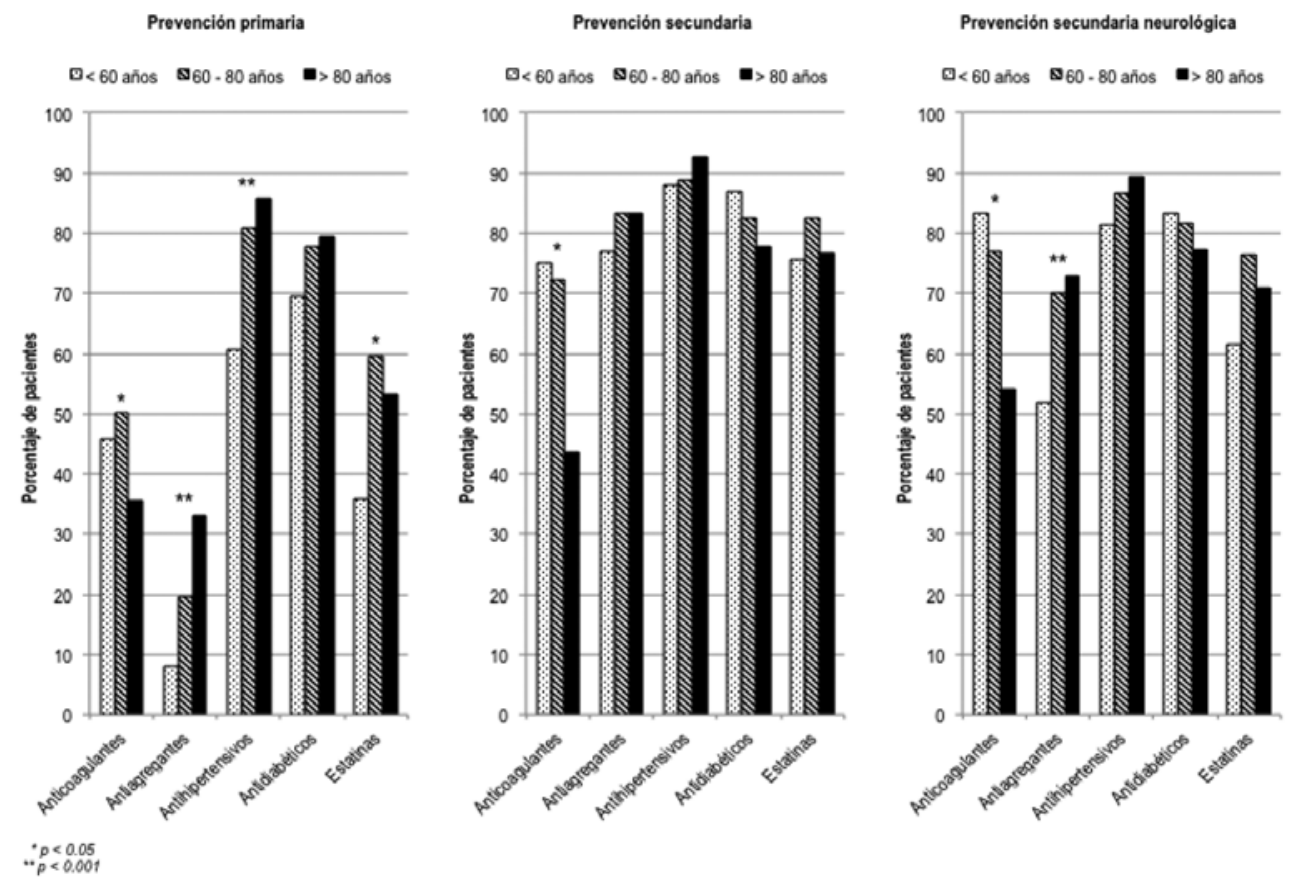

casos muy superior a los que se prescribieron en prevención primaria $(74,4 \%$ y $77,5 \%$ frente a $10 \%$; $p<0,0001)$ (Tabla I).

El grado de control de los factores de riesgo vascular también fue diferente en los tres grupos de pacientes. La hipertensión arterial, la dislipemia, el tabaquismo y la anticoagulación estuvieron significativamente peor controlados en los pacientes en prevención primaria. El mejor control de la hipertensión arterial y del tabaquismo se detectó en pacientes en prevención secundaria. En los pacientes en prevención secundaria neurológica, el control de la dislipemia y, especialmente, de la anticoagulación fue mejor ( $p=0,028$ y $p<0,0001$, respectivamente) (Tabla II).

El mal control de la hipertensión arterial ( $p=$ $0,007)$ y del enolismo $(p=0,021)$ fue mayor en los pacientes ingresados por hemorragia intracerebral; el sedentarismo $(p=0,043)$ fue superior en los pacientes con ictus isquémicos (Tabla III). En los centros que no disponían de ningún tipo de estructura asistencial para la atención a los pacientes con ictus, el porcentaje de mal control de la hipertensión arterial, de la dislipemia y de la fibrilación auricular fue mayor $(p=0,032, p<0,0001$ y $p<0,0001$, respectivamente) (Tabla IV).
El tipo de fármaco utilizado para la antiagregación plaquetaria, hipertensión arterial y dislipemia fue diferente en los tres grupos de pacientes en prevención. De forma más significativa, la aspirina y la aspirina más clopidogrel se administró más en prevención secundaria sin enfermedad cerebrovascular, mientras que el clopidogrel lo fue en pacientes con antecedentes de enfermedad cerebrovascular. Los inhibidores de la enzima conversora de angiotensina se administraron más en prevención secundaria neurológica, mientras que los betabloqueantes y los calcioantagonistas lo fueron en prevención secundaria sin enfermedad cerebrovascular previa. La atorvastatina y la sinvastatina fueron más utilizadas en el grupo de pacientes en prevención secundaria (Tabla V).

La influencia de la edad en las decisiones terapéuticas siguió un patrón similar en los tres grupos de prevención primaria, secundaria y secundaria neurológica, constituyendo el hecho más significativo la disminución de la anticoagulación en los pacientes mayores de 80 años, a pesar de que en ese grupo de edad la frecuencia de la fibrilación auricular resultó mayor $(30,6 \%$ frente a $14,1 \% ; p<0,0001)$ (Figura). 
Tabla I. Grado de cumplimiento de las medidas de prevención (en porcentaje).

\begin{tabular}{|c|c|c|c|c|}
\hline & $\begin{array}{c}\text { Prevención } \\
\text { primaria } \\
(n=3.977)\end{array}$ & $\begin{array}{l}\text { Prevención } \\
\text { secundaria } \\
(n=1.047)\end{array}$ & $\begin{array}{c}\text { Prevención } \\
\text { secundaria } \\
\text { neurológica } \\
(n=1.173)\end{array}$ & $p$ \\
\hline Fibrilación auricular & 15,6 & 20,4 & 25,1 & 0,078 \\
\hline Anticoagulantes & 43,9 & 51,4 & 60,3 & $<0,001$ \\
\hline Antiagregantes & 30,6 & 41,9 & 35,9 & $<0,001$ \\
\hline Hipertensión arterial & 62,3 & 78,3 & 74,3 & 0,133 \\
\hline Antihipertensivos & 79,3 & 86,8 & 89,8 & $<0,001$ \\
\hline Diabetes & 26,4 & 41,3 & 32,8 & 0,053 \\
\hline Antidiabéticos & 77,0 & 81,9 & 80,8 & 0,066 \\
\hline Dislipemia & 29,3 & 51,8 & 37,3 & 0,043 \\
\hline Estatinas & 56,2 & 80,3 & 73,8 & $<0,001$ \\
\hline Tabaquismo & 22,7 & 22,6 & 17,6 & 0,347 \\
\hline Medidas activas & 4,5 & 9,2 & 3,9 & 0,005 \\
\hline Enolismo & 11,7 & 10,1 & 7,8 & 0,803 \\
\hline Medidas activas & 2,7 & 3,1 & 3,0 & 0,078 \\
\hline Obesidad & 13,1 & 16,1 & 13,0 & 0,253 \\
\hline Medidas activas & 9,2 & 13,6 & 9,8 & 0,262 \\
\hline Sedentarismo & 15,0 & 23,9 & 21,9 & 0,168 \\
\hline Medidas activas & 2,2 & 3,1 & 0,6 & $<0,001$ \\
\hline $\begin{array}{l}\text { Antiagregantes en pacientes } \\
\text { sin fibrilación auricular }\end{array}$ & 19,0 & 74,4 & 77,5 & $<0,001$ \\
\hline
\end{tabular}

\section{Discusión}

El registro EPICES confirma el control insuficiente de los factores de riesgo vascular en pacientes antes y después de presentar un ictus. Este control es todavía peor en aspectos relacionados con el estilo de vida que en factores de riesgo controlables por fármacos. Sin llegar a alcanzar cifras superiores al $70 \%$ de pacientes con control inadecuado de sus factores de riesgo vascular [20-22], en nuestra serie este mal control se demuestra en un $40-50 \%$ de los pacientes, con excepción de aquellos factores de riesgo menos dependientes de los fármacos (tabaquismo, enolismo, obesidad), que alcanzan cifras superiores al $70 \%$ de los que lo presentan.
Tabla II. Percepción del neurólogo de mal control de los factores de riesgo vascular diagnosticados (en porcentaje).

\begin{tabular}{lcccc}
\hline & $\begin{array}{c}\text { Prevención } \\
\text { primaria } \\
(n=3.977)\end{array}$ & $\begin{array}{c}\text { Prevención } \\
\text { secundaria } \\
(n=1.047)\end{array}$ & $\begin{array}{c}\text { Prevención } \\
\text { secundaria } \\
\text { neurológica } \\
(n=1.173)\end{array}$ & $p$ \\
\hline $\begin{array}{l}\text { Hipertensión } \\
\text { arterial }\end{array}$ & 41,6 & 35,8 & 37,9 & 0,011 \\
\hline Diabetes & 40,4 & 44,6 & 40,0 & 0,325 \\
\hline Dislipemia & 32,6 & 27,4 & 26,1 & 0,028 \\
\hline Tabaquismo & 81,7 & 69,1 & 74,3 & $<0,001$ \\
\hline Enolismo & 86,4 & 80,0 & 77,8 & 0,058 \\
\hline Obesidad & 75,8 & 63,2 & 70,8 & 0,076 \\
\hline Sedentarismo & 13,2 & 14,8 & 19,7 & 0,058 \\
\hline Anticoagulación & 56,1 & 48,6 & 39,7 & $<0,001$ \\
\hline
\end{tabular}

Aunque el registro EPICES no identifica el colectivo médico responsable de los tres grupos asignados de prevención, se puede asumir que en los pacientes sin afectación de órganos diana, el manejo es llevado a cabo en el ámbito de la atención primaria, mientras que en los pacientes con enfermedad coronaria o cerebrovascular previa las medidas de prevención, total o parcialmente, dependen de cardiólogos o de neurólogos en el período recogido en este registro. En este supuesto, y a pesar de seguir siendo insuficiente, las medidas farmacológicas para el control de los factores de riesgo vascular son más aplicadas en prevención secundaria que en prevención primaria. Es especialmente destacable que, en prevención primaria, más del $50 \%$ de los pacientes diagnosticados de fibrilación auricular no reciben tratamiento anticoagulante y un $25,5 \%$ no reciben ningún tratamiento. Preocupa más que el porcentaje de pacientes a los que se le prescriben medidas activas para el tabaquismo, enolismo, sedentarismo u obesidad no sobrepasa el $10 \%$ en ninguno de los tres grupos de prevención.

Pese a ello, la mayor discrepancia en la intervención sobre los factores de riesgo vascular entre la prevención primaria y secundaria se refleja en la administración de antiagregantes plaquetarios en pacientes sin fibrilación auricular. A pesar de identificar más del $25 \%$ de diabéticos, más del $60 \%$ de hipertensos y el 30\% de hiperlipémicos, el porcentaje de pacientes con antiagregantes plaquetarios es in- 
Tabla III. Percepción del neurólogo de mal control de los factores de riesgo vascular diagnosticados en pacientes en prevención secundaria neurológica según el tipo de ictus (en porcentaje).

\begin{tabular}{lccc}
\hline & $\begin{array}{c}\text { Ictus } \\
\text { isquémico } \\
(n=5.429)\end{array}$ & $\begin{array}{c}\text { Hemorragia } \\
\text { intracerebral } \\
(n=768)\end{array}$ & $p$ \\
\hline Hipertensión arterial & 36,3 & 50,6 & 0,007 \\
\hline Diabetes & 34,6 & 40,3 & 0,363 \\
\hline Dislipemia & 25,0 & 26,2 & 0,544 \\
\hline Tabaquismo & 75,7 & 57,1 & 0,115 \\
\hline Enolismo & 72,2 & 85,0 & 0,021 \\
\hline Obesidad & 71,5 & 70,4 & 0,224 \\
\hline Sedentarismo & 24,5 & 18,7 & 0,043 \\
\hline
\end{tabular}

ferior al 20\%, en contra de las guías internacionales y de las opiniones de consenso [23,24].

En pacientes con enfermedad cerebrovascular previa, en gran parte controlados por neurólogos, entre un 20 y un $80 \%$ de los pacientes no controlan adecuadamente los factores de riesgo vascular; este mal control es más alarmante para el tabaquismo, el enolismo y la obesidad. Estos datos confirman el escaso tiempo que los neurólogos dedicamos a exponer al paciente los beneficios de un adecuado control dietético y de las modificaciones necesarias en los estilos de vida $[5,15,16]$. Un $40 \%$ de los pacientes neurológicos con fibrilación auricular no recibe tratamiento anticoagulante, y un porcentaje similar de los que lo reciben está mal controlado. Aunque no se conoce la eficacia en la práctica clínica habitual de los nuevos anticoagulantes, resulta razonable la esperanza de que consigan un mayor porcentaje de pacientes tratados y mejor controlados.

El control de los factores de riesgo vascular es insuficiente tanto en los pacientes con ictus isquémicos como de hemorragia intracerebral. Como cabría esperar, la hipertensión arterial y el enolismo son objetivos peor conseguidos en los pacientes de ictus que ingresan por una hemorragia intracerebral.

Es difícil explicar el inadecuado cumplimiento de las recomendaciones recogidas en las guías en relación con la demostrada eficacia del control de los factores de riesgo vascular [2]. Un registro realizado en varios países europeos en los años 19951996, 1999-2000 y 2006-2007 demuestra una ten-
Tabla IV. Percepción del neurólogo de mal control de los factores de riesgo vascular diagnosticados en pacientes en prevención secundaria neurológica según la existencia de estructuras asistenciales para la atención del ictus (en porcentaje).

\begin{tabular}{lccc}
\hline & $\begin{array}{c}\text { Alguna } \\
\text { estructura } \\
\text { asistencial } \\
(n=5.170)\end{array}$ & $\begin{array}{c}\text { Ninguna } \\
\text { estructura } \\
\text { asistencial } \\
(n=1.027)\end{array}$ & $p$ \\
\hline Hipertensión arterial & 31,4 & 42,7 & 0,032 \\
\hline Diabetes & 39,6 & 41,5 & 0,158 \\
\hline Dislipemia & 13,6 & 39,5 & $<0,0001$ \\
\hline Tabaquismo & 74,4 & 74,2 & 0,605 \\
\hline Enolismo & 77,8 & 77,8 & 0,542 \\
\hline Obesidad & 65,4 & 73,6 & 0,078 \\
\hline Sedentarismo & 18,2 & 21,5 & 0,051 \\
\hline Anticoagulación & 24,5 & 46,2 & $<0,001$ \\
\hline
\end{tabular}

dencia negativa para el control de la obesidad, el tabaquismo, la hipertensión arterial y la diabetes; tan sólo se comprueba una disminución de la hipercolesterolemia [6]. Resultados similares han sido encontrados en España durante los años 1994 y 1998 [25] en el estudio PREVESE II, y en el año 2002 en el estudio DIAPRESIC [26]. El registro EPICES no fue diseñado para un seguimiento transversal, pero el porcentaje de objetivo de buen control de los factores de riesgo vascular fue similar en los cuatro meses que se efectuó el registro a lo largo del año de seguimiento (datos no mostrados).

Aunque las estructuras asistenciales hospitalarias para la atención del ictus están dirigidas al manejo de la fase aguda, se constata que los centros que disponen de equipos, unidades o guardias de ictus presentan un mejor control en la mayoría de los factores de riesgo vascular. Sin duda, la existencia de estas estructuras facilita la formación en patología cerebrovascular y la sensibilidad de los médicos (y de la población) sobre la importancia de un tratamiento más integral.

Con la excepción de la indicación de la doble antiagregación plaquetaria en algún paciente coronario, es difícil entender las notables diferencias en los fármacos utilizados en la prevención primaria, secundaria y secundaria neurológica en ausencia de rotundas evidencias científicas. Habría que cuestionar la influencia selectiva de la industria en los di- 
Tabla V. Fármacos utilizados en la prevención de los factores de riesgo vascular (en porcentaje)

\begin{tabular}{|c|c|c|c|}
\hline & $\begin{array}{l}\text { Prevención } \\
\text { primaria } \\
(n=3.977)\end{array}$ & $\begin{array}{l}\text { Prevención } \\
\text { secundaria } \\
(n=1.047)\end{array}$ & $\begin{array}{c}\text { Prevención } \\
\text { secundaria } \\
\text { neurológica } \\
(n=1.173)\end{array}$ \\
\hline \multicolumn{4}{|l|}{ Antiagregantes ${ }^{a}$} \\
\hline Aspirina & 15,5 & 45,3 & 32,8 \\
\hline Clopidogrel & 2,3 & 17,4 & 19,1 \\
\hline Triflusal & 1,1 & 4,0 & 3,4 \\
\hline Dipiridamol & 0,1 & 0,3 & 0,3 \\
\hline Aspirina + clopidogrel & 0,2 & 7,4 & 1,7 \\
\hline Aspirina + dipiridamol & 0 & 0 & 0,2 \\
\hline \multicolumn{4}{|l|}{ Antihipertensivos ${ }^{a}$} \\
\hline IECA & 12,8 & 13,3 & 16,3 \\
\hline Diuréticos & 9,8 & 10,1 & 12,9 \\
\hline Betabloqueantes & 5,5 & 18,9 & 6,5 \\
\hline ARA II & 12,8 & 13,4 & 13,6 \\
\hline Calcioantagonistas & 9,0 & 20 & 14,4 \\
\hline Alfabloqueantes & 2,3 & 4,1 & 3,9 \\
\hline Combinaciones & 7,4 & 5,3 & 2,9 \\
\hline \multicolumn{4}{|l|}{ Estatinas $^{a}$} \\
\hline Atorvastatina & 7,4 & 28,7 & 18,5 \\
\hline Pravastatina & 2,2 & 5,4 & 3,5 \\
\hline Lovastatina & 0,8 & 1,3 & 0,6 \\
\hline Sinvastatina & 7,2 & 17,5 & 11,4 \\
\hline Fluvastatina & 0,8 & 3,1 & 1,0 \\
\hline
\end{tabular}

ARA: antagonistas de los receptores de la angiotensina; IECA: inhibidores de la enzima conversora de angiotensina. a $p<0,001$.

versos colectivos médicos responsables de la atención de los pacientes con riesgo vascular.

La disminución del porcentaje de pacientes con fibrilación auricular que recibe tratamiento anticoagulante disminuye sensiblemente en el grupo de edad con más de 80 años, independientemente del tipo de prevención. El envejecimiento de la población y la utilización de anticoagulantes más seguros razonablemente nos obligarán a no desproteger a los pacientes más añosos.

En conclusión, el registro EPICES confirma el mal control de los factores de riesgo vascular, tanto en pacientes en prevención primaria, como en los que ya han presentado enfermedad coronaria o cerebral. La existencia de estructuras asistenciales para la atención del ictus mejora el control de los pacientes con antecedentes de enfermedad cerebrovascular, aunque dista de ser el deseable. Globalmente, se demuestra que los objetivos que implican la modificación de los estilos de vida se cumplimentan peor que las medidas preventivas farmacológicas.

\section{Bibliografía}

1. Goldstein LB, Busnell CD, Adams RJ, Appel LJ, Braun LT, Chaturvedi S, et al. Guidelines for the primary prevention of stroke: a guideline for healthcare professionals from the American Heart Association/American Stroke Association. Stroke 2011; 42: 517-84.

2. Fuentes B, Gállego J, Gil-Núñez A, Morales A, Purroy F, Roquer J, et al. Guía para el tratamiento preventivo del ictus isquémico y AIT (I). Actuación sobre los factores de riesgo y estilo de vida. Neurologia 2011; Sep 3 [Epub ahead of print].

3. Clua-Espuny JL, Piñol-Moreso JL, Gil-Guillén VF, OrozcoBeltrán D, Panisello-Tafalla A, Lucas-Noll J, et al. Resultados de prevención cardiovascular primaria y secundaria en pacientes con ictus: riesgo de recurrencia y supervivencia asociada (estudio Ebrictus). Rev Neurol 2012; 54: 81-92.

4. Brotons C, Soriano N, Moral I, Rodrigo MP, Kloppe P, Rodríguez AI, et al. Randomized clinical trial to assess the efficacy of a comprehensive programme of secondary prevention of cardiovascular disease in general practice: the PREseAP study. Rev Esp Cardiol 2011; 64: 13-20.

5. Lawrence M, Kerr S, Watson H, Paton G, Ellis G. An exploration of lifestyle beliefs and lifestyle behaviour following stroke: findings from a focus group study of patients and family members. BCM Fam Pract 2010; 11: 97.

6. Kotseva K, Wood D, De Backer G, De Bacquer D, PyöräläK, Keil U; EUROASPIRE Study Group. Cardiovascular prevention guidelines in daily practice: a comparison of EUROASPIRE I, II, and III surveys in eight European countries. Lancet 2009; 373: 929-40.

7. Chen CXR, Chan SL, Law TC, Choi SK, Chan KH. Secondary prevention of stroke: an evidence-based clinical audit in the primary care. Hong Kong Med J 2011; 17: 469-77.

8. Lee S, Shafe ACE, Cowie MR. UK stroke incidence, mortality and cardiovascular risk management 1999-2008: time-trend analysis from the General Practice Research Database. BMJ Open 2011; 2: e000269.

9. Vaarjes I, Van Dis I, Grobbee DE, Bots ML. The dynamics of mortality in follow-up time after an acute myocardial infarction lower extremity arterial disease and ischemic stroke. BCM Cardiovasc Disord 2010; 10: 57.

10. Da Luz PL, Nishiyama M, Chagas ACP. Drugs and lifestyle for the treatment and prevention of coronary artery disease -comparative analysis of the scientific basis. Braz J Med Biol Res 2011; 44: 973-91.

11. Ford ES, Ajani VA, Croft JB, Critchley JA, Labarthe DR, Kottke TE, et al. Explaining the decrease in US death from coronary disease, 1980-2000. N Eng J Med 2007; 356: 2388-98.

12. Orbiagele B, Saver J, Fredier A, Suzuki S, McNair N, Dandekar R, et al. PROTECT: a coordinated stroke treatment program to prevent recurrent thromboembolic events. Neurology 2004; 63: 1217-22.

13. Hippisley-Cox J, Coupland C. Effect of combinations of drugs on all cause mortality in patients with ischaemic heart disease: nested case-control analysis. BMI 2005; 330: 1059-63.

14. Fuster V, Sanz G. Compuestos de dosis fija en la prevención secundaria de la cardiopatía isquémica. Rev Esp Cardiol 2011; 64 (Supl 2): S3-9.

15. Stroke Association. 'Nobody told me.... Highlighting the 
importance of information for stroke survivors when they leave hospital. London: Stroke Association; 2005.

16. Lawrence M, Kerr S, Watson H, Jackson J, Brownlee M. A summary of the guidance relating to four lifestyle risk factors for recurrent stroke: tobacco use, alcohol consumption diet and physical activity. Br J Neurosci Nurs 2009; 5: 471-6.

17. Park EW, Schult JK, Tudiver F, Campbell T, Becker L. Enhancing partner support to improve smoking cessation. Cochrane Database Syst Rev 2004; 3: CD002928.

18. Arias-Rivas S, Vivancos-Mora J, Castillo J, en nombre de los investigadores del registro EPICES. Epidemiología de los subtipos de ictus en pacientes hospitalizados atendidos por neurólogos: resultados del registro EPICES (I). Rev Neurol 2012; 54: 385-93.

19. Castillo J, Vivancos-Mora J, en nombre de los investigadores del registro EPICES. Tiempos de llegada al hospital y hasta la atención neurológica de pacientes con ictus agudo. Análisis de un registro multicéntrico nacional: registro EPICES (II). Rev Neurol 2012; 54: 461-7.

20. Zimetbaum PJ, Thesami A, Yu HT, Xiong Y, Liu J, Kothawala P, et al. Are atrial fibrillation patients receiving warfarin in accordance with stroke risk? Am J Med 2010; 123: 446-53.

21. Abellán-Alemani J, Ruilope-Urioste LM, Leal-Hernández M, Armario-García P, Tiberio-López G, Martell-Claros N. Control de los factores de riesgo cardiovasculares en pacientes con ictus atendidos en atención primaria en España. Estudio ICTUSCARE. Med Clin (Barc) 2011; 136: 329-35.

22. Vidal-Pérez R, Otero-Raviña F, Domínguez-López J, FabeiroRomero D, Gómez-Vázquez JL, De Blas-Abad P, et al, en representación de los investigadores del Grupo Barbanza. Características clínicas y pronóstico de pacientes con enfermedad cerebrovascular crónica (estudio ICBAR). Rev Neurol 2011; 53: 449-56.

23. Ryden L, Standl E, Bartnik M, Van den Berghe G, Betteridge J, De Boer MJ, et al. Guidelines on diabetes, pre-diabetes, and cardiovascular diseases: executive summary. The task force on diabetes and cardiovascular diseases of the European Society of Cardiology and of the European Association for the Study of Diabetes. Eur Heart J 2007; 28: 88-136.

24. Tufano A, Cimino E, Di Minno MND, Ierano P, Marrone E, Strazzullo A, et al. Diabetes mellitus and cardiovascular prevention: the role and the limitations of currently available antiplatelet drugs. Int J Vas Med 2011; 2011: 250518.

25. De Velasco JA, Cosin J, López-Sendón JL, De Teresa E, De Oya M, Sellers G. New data on secondary prevention of myocardial infarction in Spain. Results of the PREVESE II study. Rev Esp Cardiol 2002; 55: 801-9.

26. Gil-Núñez A, Vivancos J, Gabriel R. Diagnóstico y prevención secundaria del infarto cerebral en España. Estudio DIAPRESIC. Med Clin (Barc) 2008: 131: 765-9.

Compliance with the measures for preventing vascular risk factors in hospitalised patients with acute stroke. Analysis of a national multi-centre registry: EPICES registry (III)

Introduction. Pharmacological and dietary measurements, as well as life style, to control risk factors are efficient procedures to prevent cerebrovascular diseases, though their implementation in the clinics seems not optimal.

Aim. To identify the fulfillment of preventive measurements on a sample of 6197 hospitalized stroke patients, attended by neurologists.

Patients and methods. Analysis of a secondary objective of the EPICES registry. Primary prevention was considered in patients without previous record of transitory ischemic attack, stroke, coronary diseases and/or peripheral arterial disease $(n=3977)$; secondary prevention was considered in patients with record of coronary and/or peripheral arterial disease, but without record of cerebrovascular disease $(n=1047)$; neurological secondary prevention was considered in patients with a record of transitory ischemic attack and/or stroke, independently of the presence of coronary and/or peripheral arterial disease $(n=1173)$.

Results. Secondary prevention and neurological secondary prevention were more efficient than primary prevention $(p=$ 0.028 and $p<0.0001$, respectively). Control was higher in centers with some type of structure to assist stroke patients $(p<0.0001)$. Influence of age in the taking of therapeutic decisions followed a similar pattern in all three types of prevention, with a significant reduction on anticoagulation for patients older than 80 years.

Conclusion. The EPICES registry confirms the poor control of risk factors for cerebrovascular diseases. Clobally it demonstrated that the objectives that imply the modification of lifestyle are fulfilled worse than pharmacological preventive measurements.

Key words. Cerebrovascular disease. Primary prevention. Risk factors. Secondary prevention. 